Modeling, Identification and Control, Vol. 29, No. 1, 2008, pp. 29-35

\title{
A new perspective on stable inversion of non-minimum phase nonlinear systems
}

\author{
Alexey Pavlov Kristin Y. Pettersen \\ Department of Engineering Cybernetics, Norwegian University of Science and Technology, \\ N-7491 Trondheim, Norway. \\ E-mail: \{Alexey.Pavlov, Kristin.Y.Pettersen\} @itk.ntnu.no
}

\begin{abstract}
We present a new perspective on the problem of stable inversion of nonlinear non-minimum phase systems. It is based on the notion of convergent systems. The machinery of convergent systems allows us to obtain novel qualitative and quantitative conditions for solving this problem. These conditions provide insight into the dynamics behind the stable inversion problem and make it possible to treat this problem in a non-local way. Qualitatively, they cover the conditions for the stable inversion of non-minimum phase nonlinear systems previously reported in literature and allow us to solve this problem for a broader class of systems. The proposed approach is supported with a novel computational method.*
\end{abstract}

Keywords: Stable inversion, non-minimum phase systems, nonlinear systems, convergent systems.

\section{Introduction}

In output tracking control of nonlinear systems

$$
\begin{array}{ll}
\dot{x}=f(x)+g(x) u, & x \in \mathbb{R}^{n}, u \in \mathbb{R} \\
y=h(x), & y \in \mathbb{R},
\end{array}
$$

with sufficiently smooth $f(x), g(x)$ and $h(x)$, one often has to find a bounded input $u_{d}(t)$ such that system (1) with this input has a bounded solution $x_{d}(t)$ and the corresponding output equals $h\left(x_{d}(t)\right) \equiv y_{d}(t)$, where $y_{d}(t)$ is a given sufficiently smooth bounded reference output trajectory. Once such $x_{d}(t)$ and $u_{d}(t)$ are found, one can try to stabilize the desired trajectory $x_{d}(t)$ with some feedback, e.g. of the form $u=u_{d}(t)+K(x-$ $\left.x_{d}(t)\right)$.

The problem of finding the required bounded $x_{d}(t)$ and $u_{d}(t)$ - often called the stable inversion problemis conventionally approached by firstly assuming that

\footnotetext{
* This paper is based on a paper presented at the 7th IFAC Symposium on Nonlinear Control Systems, Pretoria, South Africa, 2007
}

system (1) is transformed to a normal form (see e.g. (Isidori, 1995) for the corresponding conditions)

$$
\begin{aligned}
\dot{\xi} & =p(\xi, \bar{y}, u) \\
y^{(r)} & =q(\bar{y}, \xi)+s(\bar{y}, \xi) u,
\end{aligned}
$$

where $\bar{y}:=\left(y, \dot{y}, \ldots, y^{(r-1)}\right)^{T}$ and $s(\bar{y}, \xi)$ is invertible for all $\bar{y}$ and $\xi$. For simplicity of presentation we assume that the normal form (2) is defined globally. Then from $(2 \mathrm{~b})$ we compute an input $u$ corresponding to the reference output trajectory $y_{d}(t)$ :

$$
u=s\left(\bar{y}_{d}, \xi\right)^{-1}\left(y_{d}^{(r)}-q\left(\bar{y}_{d}, \xi\right)\right)=: U\left(\xi, \bar{y}_{d}, y_{d}^{(r)}\right)
$$

where $\bar{y}_{d}:=\left(y_{d}, \dot{y}_{d}, \ldots, y_{d}^{(r-1)}\right)^{T}$. Substituting this control into $(2 \mathrm{a})$, we obtain the tracking dynamics

$$
\dot{\xi}=p\left(\xi, \bar{y}_{d}(t), U\left(\xi, \bar{y}_{d}(t), y_{d}^{(r)}(t)\right)\right)=: \bar{p}(\xi, t) .
$$

If we can find a bounded solution $\bar{\xi}(t)$ of (4), then the corresponding bounded input $u_{d}(t)$ can be computed from (3) by substituting this $\bar{\xi}(t)$ for $\xi$. The 
desired bounded solution of (2) equals $\left(\bar{\xi}^{T}(t), \bar{y}_{d}^{T}(t)\right)^{T}$ (provided that $\bar{y}_{d}(t)$ is bounded).

For non-minimum phase systems the tracking dynamics (4) are unstable, and therefore finding a bounded solution $\bar{\xi}(t)$ or even proving its existence becomes problematic. For systems with locally hyperbolic tracking dynamics the problem of finding a bounded $\bar{\xi}(t)$ was solved in (Devasia et al., 1996) with some assumptions relaxed in (Hunt and Meyer, 1997). These results were later extended to the case of time-varying systems in (Devasia and Paden, 1998), systems with uncertain parameters (Hunt et al., 1998), systems with non-hyperbolic dynamics (Devasia, 1999) and discretetime systems (Zeng and Hunt, 2000). All these papers rely on the original methods from (Devasia et al., 1996).

In this paper we propose a new approach to prove the existence of a bounded solution of the time-varying tracking dynamics (4). This approach provides novel qualitative and quantitative conditions for the stable inversion problem. These conditions give an insight into the dynamics behind the non-local stable inversion problem. Qualitatively, they include the conditions from (Devasia et al., 1996; Hunt and Meyer, 1997). Furthermore, these conditions allow us to solve the stable inversion problem for a broader class of systems that was not covered in the previous publications. In our approach we split system (4) into two subsystems which, in a certain sense, are respectively stable and unstable. The "stable" subsystem has the property that being excited by a bounded on $\mathbb{R}$ input it has a unique bounded on $\mathbb{R}$ solution and this solution is globally asymptotically stable (GAS). Systems with this property are called convergent, see (Demidovich, 1967; Pavlov et al., 2004). They are very useful for studying nonlinear systems with time-varying inputs, as shown by (Pavlov et al., 2005b,a). The "unstable" subsystem is assumed to be convergent in backward time, i.e. the unique bounded on $\mathbb{R}$ solution is GAS in backward time. We show that if the interconnection between these stable and unstable subsystems, which constitute the tracking dynamics, is weak enough, then for any $y_{d}(t)$ that is bounded on $\mathbb{R}$ together with its $r$ derivatives, the corresponding tracking dynamics have a unique solution that is bounded on $\mathbb{R}$. The weakness condition is formulated as a small gain condition. In addition to this result, we bring attention to the old yet overlooked fact due to (Demidovich, 1967) that for a time-varying nonlinear system with a unique bounded on $\mathbb{R}$ solution, like for instance the tracking dynamics (4), periodicity of the right-hand side with respect to time implies periodicity of this bounded solution. We use this fact for numerical computation of the bounded solution of the tracking dynamics.

The paper is organized as follows. In Section 2 we recall definitions of convergent systems. Section 3 contains the main results on the stable inversion problem. In Section 4 we discuss the case of the tracking dynamics with periodic inputs and propose a numerical method for solving the stable inversion problem. Section 5 contains an example and Section 6 contains conclusions.

In the paper we will use the following notations. For a vector $x,|x|$ denotes the Euclidian norm; for a matrix $M,\|M\|$ denotes the matrix norm induced by the vector norm $|\cdot|$; for a function $x(t)$ defined on $\mathbb{R}$, the norm $\|x\|_{\infty}$ is defined as $\|x\|_{\infty}:=\sup _{t \in \mathbb{R}}|x(t)| ; \bar{C}$ denotes the set of continuous functions $x(t)$ defined on $\mathbb{R}$ and satisfying $\|x\|_{\infty}<+\infty$.

\section{Convergent systems and a steady-state operator}

In this section we recall definitions of convergent systems. Consider a time-varying system

$$
\dot{x}=f(x, t), \quad x \in \mathbb{R}^{n}, \quad t \in \mathbb{R},
$$

where $f(x, t)$ is locally Lipschitz in $x$ and continuous in $t$.

Definition 1 (Pavlov et al. (2005a)) System (5) is called

- convergent if

(i) there exists a solution $\bar{x}(t)$ that is defined and bounded on $\mathbb{R}$,

(ii) $\bar{x}(t)$ is globally asymptotically stable,

- uniformly convergent if $\bar{x}(t)$ is uniformly globally asymptotically stable,

- exponentially convergent if $\bar{x}(t)$ is globally exponentially stable.

The solution $\bar{x}(t)$ is called a steady-state solution.

As shown in (Pavlov et al., 2005b), for uniformly convergent systems the steady-state solution is unique in the sense that it is the only solution that is bounded on $\mathbb{R}$. A simple example of an exponentially (and, therefore, uniformly) convergent system is a linear system $\dot{x}=A x+\phi(t)$ with a Hurwitz matrix $A$ and a bounded $\phi(t)$. An unstable counterpart of a convergent system is a system that is convergent in backward time.

Definition 2 System (5) is called (uniformly, exponentially) convergent in backward time if the system $\dot{x}=-f(x,-t)$ is (uniformly, exponentially) convergent. 
Systems that are convergent in backward time also have a bounded $\mathbb{R}$ solution (unique for the uniformly convergent case), but this solution is "anti-stable"globally asymptotically stable in backward time. We will also refer to this solution as a steady-state solution.

When talking about systems of the form

$$
\dot{x}=f(x, w, t), \quad x \in \mathbb{R}^{n}, w \in \mathbb{R}^{m}, t \in \mathbb{R}
$$

with input $w$, we say that system (6) is (uniformly, exponentially) convergent (in backward time) for the class of inputs $\bar{C}$, if for any $w \in \bar{C}$ the system $\dot{x}=$ $f(x, w(t), t)$ is (uniformly, exponentially) convergent (in backward time). The steady-state solution corresponding to the input $w(t)$ is denoted by $\bar{x}_{w}(t)$. Since for a uniformly convergent (in backward time) system (6) for any input $w \in \bar{C}$ there is a unique steady-state solution $\bar{x}_{w}(t)$, we can define the so-called steady-state operator.

Definition 3 The operator $\mathcal{F}: \bar{C} \rightarrow \bar{C}$ defined as $\mathcal{F} w(t):=\bar{x}_{w}(t)$ is called a steady-state operator of the uniformly convergent (in backward time) system (6).

The next theorem contains a technical result that will be used in the next section.

Theorem 1 Consider system (6) with $f(x, w, t)$ being continuous in $w, t$ and $C^{1}$ in $x$. Suppose there exists a scalar $\alpha>0$ such that

$$
\frac{\partial f}{\partial x}(x, w, t)+\frac{\partial f^{T}}{\partial x}(x, w, t) \leq-2 \alpha I,
$$

for all $x, w$ and $t$, and that for any $r>0$ it holds that

$$
\sup _{|w| \leq r, t \in \mathbb{R}}|f(0, w, t)|<+\infty \text {. }
$$

Then system (6) is exponentially convergent for the class of inputs $\bar{C}$. Moreover, if

$$
\left\|\frac{\partial f}{\partial w}(x, w, t)\right\| \leq L, \quad \forall x, w, t,
$$

for some constant $L>0$, then the corresponding steadystate operator $\mathcal{F}$ is Lipschitz continuous, i.e.

$$
\left\|\mathcal{F} w_{1}-\mathcal{F} w_{2}\right\|_{\infty} \leq \gamma\left\|w_{1}-w_{2}\right\|_{\infty}
$$

with the Lipschitz constant $\gamma=L / \alpha$.

The proof of the exponential convergence can be found in (Demidovich, 1967; Pavlov et al., 2004). The proof of (10) can be found in Appendix.

Remark 1 For simplicity of the presentation, in Theorem 1 we use condition (7) instead of the more general condition

$$
P \frac{\partial f}{\partial x}(x, w, t)+\frac{\partial f^{T}}{\partial x}(x, w, t) P \leq-Q,
$$

for some $P=P^{T}>0$ and $Q=Q^{T}>0$. System (6) satisfying (11) can be transformed to a system satisfying condition (7) by means of the coordinate transformation $\tilde{x}=P^{1 / 2} x$, see (Pavlov et al., 2002).

\section{Main results}

In this section we formulate conditions on the tracking dynamics (4) under which there is a unique bounded on $\mathbb{R}$ solution $\bar{\xi}(t)$. As discussed in Section 2, if the tracking dynamics (4) are uniformly convergent (like in the case of minimum phase linear systems), or uniformly convergent in backward time, then system (4) has a unique bounded on $\mathbb{R}$ solution. Next we consider the case when (4) can be decomposed (after, possibly, a coordinate transformation) into a series connection of two systems:

$$
\begin{aligned}
& \dot{\eta}=F(\eta, t), \\
& \dot{\zeta}=G(\zeta, \eta, t) .
\end{aligned}
$$

If system (12a) is uniformly convergent and (12b) with $\eta$ as input is uniformly convergent in backward time for the class of inputs $\bar{C}$, one can easily verify that the bounded on $\mathbb{R}$ solution of (12) is unique and it equals $\left(\bar{\eta}^{T}(t), \bar{\zeta}_{\bar{\eta}}^{T}(t)\right)^{T}$, where $\bar{\eta}(t)$ is the steady-state solution of $(12 \mathrm{a})$ and $\bar{\zeta}_{\bar{\eta}}(t)$ is the steady-state solution of $(12 \mathrm{~b})$ corresponding to the input $\bar{\eta}(t)$. If system (12a) is allowed to depend on $\zeta$, i.e.

$$
\begin{aligned}
& \dot{\eta}=F(\eta, \zeta, t), \\
& \dot{\zeta}=G(\zeta, \eta, t),
\end{aligned}
$$

then the conditions for the existence and uniqueness of a bounded on $\mathbb{R}$ solution become more involved.

Theorem 2 Consider system (13). Suppose a) system (13a) with $\zeta$ as input is uniformly convergent for the class of inputs $\bar{C}$ with the corresponding steady-state operator $\mathcal{F}$ being Lipschitz continuous with a Lipschitz constant $\gamma_{s}$;

b) system (13b) with $\eta$ as input is uniformly convergent in backward time for the class of inputs $\bar{C}$ with the corresponding steady-state operator $\mathcal{G}$ being Lipschitz continuous with a Lipschitz constant $\gamma_{u}$. If

$$
\gamma_{u} \gamma_{s}<1
$$

then system (13) has a unique bounded on $\mathbb{R}$ solution $\left(\bar{\eta}^{T}(t), \bar{\zeta}^{T}(t)\right)^{T}$.

Proof. See Appendix.

This result provides qualitative conditions on the tracking dynamics that guarantee the existence of a bounded solution. Roughly speaking, these conditions 
may be called conditions of non-locally hyperbolic dynamics: the system is decomposed into a convergent in forward time dynamics (the "stable" part) and convergent in backward time dynamics (the "unstable" part). When considered separately from each other, each subsystem has the property that being excited by a bounded input it has a unique bounded on $\mathbb{R}$ solution. The small gain condition (14) guarantees that the influence of these two subsystems on each other does not destroy this property for the interconnected system. Uniting Theorems 1 and 2 we directly obtain the following result.

Theorem 3 Let the right-hand side of (13) be continuous in $t$ and $C^{1}$ with respect to $\eta$ and $\zeta$. Suppose there exist constants $\alpha_{s}>0$ and $\alpha_{u}>0$ such that

$$
\begin{gathered}
\frac{\partial F}{\partial \eta}(\eta, \zeta, t)+\frac{\partial F^{T}}{\partial \eta}(\eta, \zeta, t) \leq-2 \alpha_{s} I, \\
\frac{\partial G}{\partial \zeta}(\zeta, \eta, t)+\frac{\partial G^{T}}{\partial \zeta}(\zeta, \eta, t) \geq 2 \alpha_{u} I,
\end{gathered}
$$

for all $\eta, \zeta$ and $t$; and that for any $r>0$ it holds that

$$
\begin{aligned}
& \sup _{|\zeta| \leq r, t \in \mathbb{R}}|F(0, \zeta, t)|<+\infty, \\
& \sup _{|\eta| \leq r, t \in \mathbb{R}}|G(0, \eta, t)|<+\infty,
\end{aligned}
$$

and there exist constants $L_{s}, L_{u}$ such that

$$
\begin{gathered}
\left\|\frac{\partial F}{\partial \zeta}(\eta, \zeta, t)\right\| \leq L_{s}, \\
\left\|\frac{\partial G}{\partial \eta}(\zeta, \eta, t)\right\| \leq L_{u},
\end{gathered}
$$

for any $\eta, \zeta$ and $t$. If

$$
\frac{L_{s} L_{u}}{\alpha_{s} \alpha_{u}}<1,
$$

then system (13) has a unique bounded on $\mathbb{R}$ solution $\left(\bar{\eta}^{T}(t), \bar{\zeta}^{T}(t)\right)^{T}$.

Proof. According to Theorem 1, conditions (15), (17) and (19) guarantee that system (13a) is exponentially convergent for the class of inputs $\bar{C}$ with the corresponding steady-state operator having the Lipschitz constant $\gamma_{s}=L_{s} / \alpha_{s}$. In the same way, conditions (16) (18) and (20) guarantee that system (13b) is exponentially convergent in backward time with the steadystate operator having the Lipschitz corresponding constant $\gamma_{u}=L_{u} / \alpha_{u}$. Application of Theorem 2 concludes the proof. $\square$

Using Theorem 3 one can prove the following corollary. Its proof is omitted here due to space limitations.

\section{Corollary 1 Consider the system}

$$
\dot{\xi}=A \xi+H(\xi, t), \quad \xi \in \mathbb{R}^{p}, t \in \mathbb{R},
$$

with the matrix A having no eigenvalues on the imaginary axis and the nonlinearity $H(\xi, t)$ being continuous with respect to $t$ and $C^{1}$ with respect to $\xi$. There exists a number $\epsilon>0$, depending only on the matrix $A$, such that if

$$
\begin{gathered}
\left\|\frac{\partial H}{\partial \xi}(\xi, t)\right\| \leq \epsilon \quad \forall \xi, t \\
\sup _{t \in \mathbb{R}}|H(0, t)|<+\infty
\end{gathered}
$$

then system (22) has a unique bounded on $\mathbb{R}$ solution.

As follows from this corollary, if a nonlinear timevarying system is sufficiently approximated by a linear hyperbolic time invariant system in the sense that the Jacobian of the nonlinear system is close enough to a constant matrix having no eigenvalues on the imaginary axis, then this nonlinear system has a unique bounded on $\mathbb{R}$ solution. For smooth systems one can obtain such an approximation near the origin, as it is used in (Devasia et al., 1996). Therefore, one can say that qualitatively this corollary is a global counterpart of the (local) results from (Devasia et al., 1996; Hunt and Meyer, 1997).

\section{Periodic tracking dynamics and computational issues}

In practice it is not enough to prove the existence of a bounded trajectory of the tracking dynamics. One also needs to have reliable numerical methods for computing this trajectory. In this section we show how this problem can be reduced to a problem of computing periodic solutions of a nonlinear system - the problem for which numerical algorithms already exist, see e.g. (Parker and Chua, 1989).

Consider the tracking dynamics (4), but this time we explicitly express the dependency of the right-hand side on the desired output trajectory $y_{d}(t)$ :

$$
\dot{\xi}=\bar{p}\left(\xi, w_{d}(t)\right)
$$

with the input $w_{d}(t)=\left(y_{d}(t), \dot{y}_{d}(t), \ldots, y_{d}^{(r)}(t)\right)^{T}$. Let us first consider the case of the periodic sufficiently smooth $y_{d}(t)$, which implies that $w_{d}(t)$ is also periodic.

Lemma 1 (Demidovich (1967)) Consider system (24). Suppose $w_{d}(t+T) \equiv w_{d}(t)$ for some $T>0$ and (24) has a unique solution $\bar{\xi}(t)$ that is bounded on $\mathbb{R}$. Then $\bar{\xi}(t+T) \equiv \bar{\xi}(t)$. 
According to this lemma, the desired bounded solution of the tracking dynamics is also periodic with the same period as $y_{d}(t)$. Hence this solution can be found using one of the numerical techniques for computing periodic solutions of nonlinear systems, for example using the shooting method, see e.g. (Parker and Chua, 1989).

In practice non-periodic output reference trajectories $y_{d}(t)$ are usually specified on some finite time interval $[0, T]$. We can always extend the corresponding $w_{d}(t)$ to $\mathbb{R}$ such that the extended input $w_{d}^{\text {ext }}(t)$ is continuous and periodic. Then we can find the unique periodic solution $\bar{\xi}(t)$ corresponding to this $w_{d}^{e x t}(t)$ using, for example the shooting method. By the construction of $w_{d}^{\text {ext }}(t)$, on the time interval $[0, T]$ this bounded solution $\bar{\xi}(t)$ will correspond to the original $w_{d}(t)$.

\section{Example}

Consider the tracking dynamics given by

$$
\begin{aligned}
& \dot{\eta}=-\eta-\eta^{3}+a \zeta+\psi_{1}\left(w_{d}(t)\right)=: F(\eta, \zeta, t) \\
& \dot{\zeta}=\zeta+\zeta^{5}+b \eta+\psi_{2}\left(w_{d}(t)\right)=: G(\zeta, \eta, t)
\end{aligned}
$$

where $\zeta \in \mathbb{R}, \eta \in \mathbb{R}, \psi_{1}(w)$ and $\psi_{2}(w)$ are arbitrary continuous functions and $w_{d}(t)$ is a bounded on $\mathbb{R}$ continuous input determined by some reference output trajectory. Let us show that this system satisfies the conditions of Theorem 3. The partial derivative of the right-hand side of $(25 \mathrm{a})$ with respect to $\eta$ equals $\frac{\partial F}{\partial \eta}=-1-3 \eta^{2}$. Hence

$$
\frac{\partial F}{\partial \eta}+\frac{\partial F^{T}}{\partial \eta}=-2-6 \eta^{2} \leq-2
$$

i.e. condition (15) is satisfied with $\alpha_{s}=1$. Condition (17) holds for any bounded on $\mathbb{R} w_{d}(t)$. Finally, condition (19) holds with $L_{s}=|a|$, since $\frac{\partial F}{\partial \zeta}=a$. In the same way, conditions (16), (18) and (20) hold for system (25b) with $\alpha_{u}=1$ and $L_{u}=|b|$. Applying Theorem 3 we conclude that if $|a b|<1$ (condition (21)), then for an arbitrary continuous input $w_{d}(t)$ that is bounded on $\mathbb{R}$, system (25) has a unique bounded on $\mathbb{R}$ solution.

Note that the right-hand side of (25) is not Lipschitzsomething that would be required in order to prove, for an arbitrary bounded $w_{d}(t)$, the existence of a bounded on $\mathbb{R}$ solution using the techniques of (Devasia et al., 1996) or (Hunt and Meyer, 1997). This demonstrates an advantage of the method proposed in this paper, which makes it applicable to a broader class of nonlinear systems.

\section{Conclusions}

We have presented a new approach to solving the (global) problem of stable inversion of non-minimum phase nonlinear systems. This approach is based on the notion of convergent systems. It provides a new insight into the dynamics behind this problem and it gives sufficient conditions that qualitatively cover the existing results and allow us to solve the stable inversion problem for a broader class of nonlinear systems. Furthermore, we have proposed a numerical method for solving the stable inversion problem based on reducing this problem to finding periodic solutions of a nonlinear system.

\section{Appendix}

Proof of Theorem 1. Let $\bar{x}(t):=\mathcal{F} w(t)$ and $\tilde{x}(t):=$ $\mathcal{F}(w+\Delta w)(t)$ denote the steady-state solutions of system (6) corresponding to the inputs $w(t)$ and $w(t)+$ $\Delta w(t)$, respectively. Consider the system

$$
\begin{aligned}
\Delta \dot{x}= & f(\bar{x}(t)+\Delta x, w(t)+\Delta w, t) \\
& -f(\bar{x}(t), w(t), t)
\end{aligned}
$$

with $\Delta w$ as input. The difference $\overline{\Delta x}(t):=\tilde{x}(t)-$ $\bar{x}(t)$ is the only solution of (26) that is bounded on $\mathbb{R}$ (because, by definition, both $\bar{x}(t)$ and $\tilde{x}(t)$ are the only bounded on $\mathbb{R}$ solutions of system (6) with inputs $w(t)$ and $w(t)+\Delta w(t)$, respectively).

Consider the function $V(\Delta x):=\frac{1}{2}|\Delta x|^{2}$. Its derivative along solutions of (26) equals

$$
\begin{aligned}
& \dot{V}=\Delta x^{T}(f(\bar{x}+\Delta x, w+\Delta w, t)-f(\bar{x}, w, t)) \\
& =\Delta x^{T}(f(\bar{x}+\Delta x, w+\Delta w, t)-f(\bar{x}, w+\Delta w, t)) \\
& +\Delta x^{T}(f(\bar{x}, w+\Delta w, t)-f(\bar{x}, w, t)) .
\end{aligned}
$$

It is shown in (Pavlov et al., 2004) that condition (7) implies

$$
\begin{aligned}
& \Delta x^{T}(f(\bar{x}+\Delta x, w+\Delta w, t)-f(\bar{x}, w+\Delta w, t)) \\
& \leq-\alpha|\Delta x|^{2} .
\end{aligned}
$$

The second component in (27) can be estimated as

$$
\begin{aligned}
& \Delta x^{T}(f(\bar{x}, w+\Delta w, t)-f(\bar{x}, w, t)) \\
& \leq|\Delta x||f(\bar{x}, w+\Delta w, t)-f(\bar{x}, w, t)| \\
& \leq|\Delta x|\left\|\frac{\partial f}{\partial w}(\bar{x}, \omega, t)\right\||\Delta w| \\
& \leq|\Delta x| L|\Delta w| .
\end{aligned}
$$

The estimate (29a) holds for some $\omega$ lying on the line segment $(w, w+\Delta w)$. In $(29 \mathrm{~b})$ we have used condition 
(9). Substituting the estimates (28) and (29) into (27), we obtain

$$
\dot{V} \leq-\alpha|\Delta x|^{2}+|\Delta x| L|\Delta w|
$$

Hence $\dot{V}<0$ for $|\Delta x|>|\Delta w| L / \alpha$. From this inequality, following the reasoning of Theorem 4.18 from (Khalil, 2002), one can show that any bounded on $\mathbb{R}$ solution of $(26)$ - in particular $\overline{\Delta x}(t)$ - lies inside the set $|\Delta x| \leq \frac{L}{\alpha}\|\Delta w\|_{\infty}$. Therefore,

$$
\|\overline{\Delta x}\|_{\infty}=\|\mathcal{F}(w+\Delta w)-\mathcal{F} w\|_{\infty} \leq \gamma\|\Delta w\|_{\infty},
$$

with $\gamma=L / \alpha$, i.e. (10) holds.

Proof of Theorem 2. Let us first show that there exist numbers $\sigma>0$ and $r>0$ such that $\mathcal{F}: B_{r} \rightarrow B_{\sigma}$ and $\mathcal{G}: B_{\sigma} \rightarrow B_{r}$, where $B_{r}:=\left\{\zeta \in \bar{C}:\|\zeta\|_{\infty} \leq r\right\}$ and $B_{\sigma}:=\left\{\eta \in \bar{C}:\|\eta\|_{\infty} \leq \sigma\right\}$. Notice that

$$
\begin{aligned}
\|\mathcal{F} \zeta\|_{\infty} & \leq\|\mathcal{F} \zeta-\mathcal{F} 0\|_{\infty}+\|\mathcal{F} 0\|_{\infty} \\
& \leq \gamma_{s}\|\zeta\|_{\infty}+\|\mathcal{F} 0\|_{\infty} .
\end{aligned}
$$

For $\zeta \in B_{r}$ we obtain

$$
\|\mathcal{F} \zeta\|_{\infty} \leq \gamma_{s} r+\|\mathcal{F} 0\|_{\infty}
$$

In the same way, we obtain that for $\eta \in B_{\sigma}$,

$$
\|\mathcal{G} \eta\| \leq \gamma_{u} \sigma+\|\mathcal{G} 0\|_{\infty}
$$

Choose

$$
\begin{aligned}
\sigma & :=\frac{\|\mathcal{F} 0\|_{\infty}+\gamma_{s}\|\mathcal{G} 0\|_{\infty}}{1-\gamma_{s} \gamma_{u}}, \\
r & :=\frac{\|\mathcal{G} 0\|_{\infty}+\gamma_{u}\|\mathcal{F} 0\|_{\infty}}{1-\gamma_{s} \gamma_{u}} .
\end{aligned}
$$

Due to the small gain condition (14), $r>0$ and $\sigma>0$. Substituting these $r$ and $\sigma$ into (32) and (33), we obtain $\|\mathcal{F} \zeta\|_{\infty} \leq \sigma$ and $\|\mathcal{G} \eta\|_{\infty} \leq r$ for $\zeta \in B_{r}$ and $\eta \in B_{\sigma}$, i.e. $\mathcal{F}: B_{r} \rightarrow B_{\sigma}$ and $\mathcal{G}: B_{\sigma} \rightarrow B_{r}$.

Next, consider the mapping $\mathcal{H}:=\mathcal{G} \circ \mathcal{F}$. By the reasoning presented above, we see that $\mathcal{H}$ maps $B_{r}$ into $B_{r}$. One can easily check that $B_{r}$ is a complete metric space with the metric induced by the norm $\|\cdot\|$. Moreover,

$$
\begin{aligned}
\left\|\mathcal{H} \zeta_{1}-\mathcal{H} \zeta_{2}\right\|_{\infty} & =\left\|\mathcal{G} \circ \mathcal{F} \zeta_{1}-\mathcal{G} \circ \mathcal{F} \zeta_{2}\right\|_{\infty} \\
& \leq \gamma_{u}\left\|\mathcal{F} \zeta_{1}-\mathcal{F} \zeta_{2}\right\|_{\infty} \\
& \leq \gamma_{u} \gamma_{s}\left\|\zeta_{1}-\zeta_{2}\right\|_{\infty} .
\end{aligned}
$$

Due to the small gain condition (14), the mapping $\mathcal{H}$ is a contraction. Applying the Banach fixed point theorem (see, e.g. (Kreyszig, 1978)), we conclude that in the set $B_{r}$ there is a unique function $\bar{\zeta}$ satisfying the equation $\bar{\zeta}=\mathcal{H} \bar{\zeta}$. In fact, $\bar{\zeta}$ is the only solution of this equation not only in $B_{r}$, but in $\bar{C}$. Namely, suppose $\bar{\zeta}_{1}$ and $\bar{\zeta}_{2}$ are two such solutions. Then

$$
\left\|\bar{\zeta}_{1}-\bar{\zeta}_{2}\right\|_{\infty}=\left\|\mathcal{H} \bar{\zeta}_{1}-\mathcal{H} \bar{\zeta}_{2}\right\|_{\infty} \leq \gamma_{u} \gamma_{s}\left\|\bar{\zeta}_{1}-\bar{\zeta}_{2}\right\|_{\infty}
$$

Since $\gamma_{u} \gamma_{s}<1$ this inequality implies that $\left\|\bar{\zeta}_{1}-\bar{\zeta}_{2}\right\|_{\infty}=$ 0 , which proves uniqueness of such $\bar{\zeta}$.

Now let's have a look at the meaning of the mapping $\mathcal{H}$. Consider the system

$$
\begin{aligned}
& \dot{\eta}=F(\eta, \tilde{\zeta}, t) \\
& \dot{\zeta}=G(\zeta, \eta, t)
\end{aligned}
$$

Consider some input $\tilde{\zeta} \in \bar{C}$. Then $\mathcal{F} \tilde{\zeta}(t)$ is the steadystate solution of system (35a). Substitute this $\mathcal{F} \tilde{\zeta}(t)$ for $\eta$ in (35b). The corresponding steady-state solution of system $(35 \mathrm{~b})$ equals $\mathcal{H} \tilde{\zeta}(t)$. Therefore, $\bar{\zeta}(t)-$ the solution of the equation $\bar{\zeta}=\mathcal{H} \bar{\zeta}$-is such that the pair $\bar{\eta}(t):=\mathcal{F} \bar{\zeta}(t)$ and $\bar{\zeta}(t)$ form a bounded on $\mathbb{R}$ solution of (13). This completes the proof. $\square$

\section{References}

Demidovich, B. Lectures on stability theory (in Russian). Nauka, Moscow, 1967.

Devasia, S. Approximated stable inversion for nonlinear systems with nonhyperbolic internal dynamics. IEEE Trans. Automatic Control, 1999. 44:14191424.

Devasia, S., Chen, D., and Paden, B. Nonlinear inversion-based output tracking. IEEE Trans. Automatic Control, 1996. 41:930-942.

Devasia, S. and Paden, B. Stable inversion for nonlinear nonminimum-phase time-varying systems. IEEE Trans. Automatic Control, 1998. 43:283-288.

Hunt, L., Ramakrishna, V., and Meyer, G. Stable inversion and parameter variations. Systems \& Control Letters, 1998. 34:203-207.

Hunt, L. R. and Meyer, G. Stable inversion for nonlinear systems. Automatica, 1997. 33:1549-1554.

Isidori, A. Nonlinear control systems, 3rd ed. SpringerVerlag, London, 1995.

Khalil, H. Nonlinear systems, 3rd ed. Prentice Hall, Upper Saddle River, 2002.

Kreyszig, E. Introductory Functional Analysis with Applications. John Wiley \& Sons, New York, 1978.

Parker, T. and Chua, L. Practical numerical algorithms for chaotic systems. Springer-Verlag, New York, 1989.

Pavlov, A., Pogromsky, A., van de Wouw, N., and Nijmeijer, H. Convergent dynamics, a tribute to Boris Pavlovich Demidovich. Systems and Control Letters, 2004. 52:257-261. 
Pavlov, A., van de Wouw, N., and Nijmeijer, H. Convergent systems and the output regulation problem. In Proc. of IEEE Conf. Decision and Control. 2002 pages 2681-2686.

Pavlov, A., van de Wouw, N., and Nijmeijer, H. Convergent systems: analysis and design. In Control and Observer Design for Nonlinear Finite and Infinite Dimensional Systems, pages 131-146. Springer, 2005a.

Pavlov, A., van de Wouw, N., and Nijmeijer, H. Uniform output regulation of nonlinear systems: a convergent dynamics approach. Birkhäuser, Boston, 2005b.

Zeng, G. and Hunt, L. Stable inversion of nonlinear discrete-time systems. IEEE Trans. Automatic Control, 2000. 45:1216-1220. 